\title{
Canonical and Singular Propagation of Ultrashort Pulses in a Nonlinear Medium
}

\author{
Karl Glasner, ${ }^{1}$ Miroslav Kolesik, ${ }^{2,3,4}$ Jerome V. Moloney, ${ }^{1,2,3}$ and Alan C. Newell ${ }^{1,2}$ \\ ${ }^{1}$ Department of Mathematics, University of Arizona, Tucson, AZ 85721-0089, USA \\ ${ }^{2}$ Arizona Center for Mathematical Sciences, University of Arizona, Tucson, AZ 85721-0089, USA \\ ${ }^{3}$ College of Optical Sciences, University of Arizona, Tucson, AZ 85721, USA \\ ${ }^{4}$ Department of Physics, Constantine the Philosopher University, Nitra 94974, Slovakia
}

Correspondence should be addressed to Jerome V. Moloney, jml@acms.arizona.edu

Received 14 May 2011; Revised 13 July 2011; Accepted 14 July 2011

Academic Editor: Shunichi Sato

Copyright (C) 2012 Karl Glasner et al. This is an open access article distributed under the Creative Commons Attribution License, which permits unrestricted use, distribution, and reproduction in any medium, provided the original work is properly cited.

We examine the two types of singular behaviors of ultrashort pulses in a nonlinear medium, pulse steepening if the weak longitudinal dispersion is normal and collapse if it is anomalous. Connections with analogous behaviors of wave packets of almost monochromatic waves in strongly dispersive media are discussed.

\section{Introduction}

The drive to achieve ever higher local intensities with finite amounts of energy is the main reason that has led to much interest into the propagation characteristics of ultrashort pulses whose spectrum is broad, and whose widths are no more than several wavelengths of light. A second reason is that they exhibit novel singular behaviors, behaviors which are worth contrasting with those of "rogue" waves, familiar from ocean contexts and the "collapses" associated with the envelopes of almost monochromatic waves associated with singular solutions of equations of nonlinear Schrödinger type. A third reason is the ongoing program of attempting to create suitable conditions under which one might effectively propagate high intensity short pulses over long distances through gases such as the atmosphere.

Our aim in this short paper is to provide a unified mathematical description via a nonlinear partial differential equation that captures both type of singularity while avoiding the usual envelope approximations utilized in the nonlinear optics literature. Many of the ideas and some of the equation derivations, albeit in different formats, have appeared already in the literature; for example, in the recent works of Balakin et al. (BLMS; [1]) which in turn were informed by results going back to the eighties $[2,3]$; the papers of Alterman and Rauch [4] and Schaffer and Wayne
[5], and the results of Kolesik and Moloney [6]. Indeed, in many cases the authors appeared to be unaware of each other's results. Our goals here are as follows.

We want to show that the series of governing equations (UPPE, an acronym for unidirectional pulse propagation equation) derived by Kolesik and Moloney [6] directly from Maxwell's equations using a Fourier decomposition of the electromagnetic fields can be reduced by asymptotic analysis to an equation for the dominant electric field in which all wavelength (short, $\mu \mathrm{m}$ ) scales have been removed, essentially by averaging over wavelength scales. We call the reduced equation RUPPE. In RUPPE, the electric field varies over distances associated with nonlinearity, diffraction, dispersion, and attenuation. The Fourier representation is particularly valuable because it shows clearly the circumstances in which nonlinearity has long distance cumulative, order one, effects. It will let us see, for example, how, if the pulse is broadband, dispersion in the direction of propagation (longitudinal dispersion) must necessarily be weak. This contrasts with the situation in which the pulse is almost monochromatic with a narrowband spectrum, in which case, with strong dispersion, the propagation equation will be more of nonlinear Schrödinger type.

We show how, by taking the inverse Fourier transform of RUPPE, we arrive at the modified Kadomtsev-Petviashvili 
equation (MKPI) of type I which is closely related to the equation derived in the early eighties by Kuznetsov for acoustic waves [2], for which equation with cubic nonlinearity, various "blowup" results were found by Turitsyn and Falkovich [3], and which is the main equation used by BLMS in their analysis. If $E_{0}\left(x, y, z, \tau=t-\left(n_{0} / c\right) z\right)$ is the dominant (either linearly or azimuthally polarized) component of the electric field, then the MKPI equation in nondimensional form is

$$
\begin{aligned}
& \frac{\partial}{\partial \tau}\left(\frac{\partial E_{0}}{\partial z}+\frac{z_{0} E_{0}^{2}}{z_{\mathrm{NL}}} \frac{\partial E_{0}}{\partial \tau}-\frac{z_{0} s}{z_{\text {disp }}} \frac{\partial^{3} E_{0}}{\partial \tau^{3}}-\frac{z_{0}}{z_{\text {att }}} \frac{\partial^{2} E_{0}}{\partial \tau^{2}}\right) \\
& \quad=\frac{z_{0}}{z_{\text {diff }}} \delta^{2} E_{0}
\end{aligned}
$$

Equation (1) shows how the right going Riemann invariant $E_{0}$ of the underlying wave equation is deformed over long distances by a combination of nonlinear, diffraction, dispersion, and attenuation influences.

In $(1), z_{\mathrm{NL}}, z_{\text {disp }}, z_{\mathrm{att}}$, and $z_{\text {diff }}$ are the nonlinear, dispersion, attenuation, and diffraction distances defined by

$$
\begin{gathered}
z_{\mathrm{NL}}=\frac{n_{0} c t_{0}}{3 \pi \chi^{(3)} e_{0}^{2}}, \quad z_{\text {disp }}=\frac{2 n_{0} c t_{0}}{|B|}, \\
z_{\mathrm{att}}=\frac{2 n_{0} c t_{0}}{A}, \quad z_{\mathrm{diff}}=\frac{2 n_{0} L^{2}}{c t_{0}}
\end{gathered}
$$

where $s=\operatorname{sgn} B$ and $\chi^{(3)} e_{0}^{2}=(8 / 3) n_{0} n_{2} I$ is related to the nondimensional pulse intensity, $n_{0}, n_{2}$ the linear and nonlinear refractive indices, $t_{0}$ pulse width, $L$ beam width and $z_{0}$ is the shortest distance (usually $z_{\mathrm{NL}}$ or $z_{\text {diff }}$ over which $E_{0}$ changes. The nondimensional coefficients $B$ and $A$ describe how the real and imaginary parts of the linear susceptibility deviate from constant values. The operator $\delta^{2}$ is the Laplacian $\partial^{2} / \partial x^{2}+\partial^{2} / \partial y^{2}=\partial^{2} / \partial r^{2}+(1 / r)(\partial / \partial r)$ if the field is asymmetric and linearly polarized and $\partial^{2} / \partial r^{2}+$ $(1 / r)(\partial / \partial r)-1 / r^{2}$ for the azimuthally polarized case.

We explore singular behavior in (1). By singular behavior, we mean that there will be a finite time singularity, namely unbounded growth tending to infinity in a finite time, in either the amplitude or the slope of the electric field. Dispersion, as indeed does dissipation, regularizes the singularity in that either the amplitude or slope becomes very large before the unbounded growth gets arrested. For example, if the singularity is in the slope, the pulse steepens until the dispersion or dissipation terms become sufficiently large so as to bring about a balance between the nonlinear steepening effect, manifested by the time derivative of the electric field cubed and the dissipation or dispersion. If the singularity is in the amplitude, then the arrest is a bit more subtle and involves the property that in two transverse space dimensions the collapsing pulse carries exactly the critical amount of energy (power) required to affect collapse. Any loss, through dissipation or radiative dispersion will have the effect of bringing the power below critical, and then the collapse slowly radiates away in waves. The point of the paper is to emphasize that because dispersion and dissipation are small when applied to the original pulse shape, the pulse will evolve a long way towards the collapse state before being arrested, as indeed our numerics show.

We find that there are two distinct types of singular behavior exhibited by solutions of (1). If the dispersion is normal, namely $B>0$, then the evolution of the electric field is dominated by the first two terms. The leading part of the pulse (it is the rear part if viewed from a fixed frame of reference) steepens until either dispersion causes the steepening front to form oscillations, or diffusion causes the front to develop a shock. This is the case studied by BLMS [1]. We make a very important point. Unlike KPI, namely where the nonlinearity is quadratic, the MKPI whose behavior in the longitudinal direction satisfies the modified Korteweg-de Vries equation, the difference in signs of nonlinearity and dispersion is crucial. For $B>0$, the oscillations, which the steepening front produces, never become solitons. They are purely radiative and eventually disperse. Indeed what we show is that they behave rather like normal dispersion does in arresting the collapse of the two dimensional nonlinear Schrödinger equation discussed by us in the mid nineties [7]. Thus, the energy of the oscillating waves spreads into the transverse direction by four-wave resonant interactions. This behavior is clearly seen in our simulations and was extensively reported in the simulations of Kolesik and Moloney [6]. On the other hand, if dispersion is anomalous, $B<0$, then solitons do form and destabilize into local collapses with an amplitude singularity when diffractive effects become important. By diffractive effects, we are referring to the dispersion brought about by the diffraction term which causes pulses whose carrier wavevector is not quite along the $\mathrm{z}$ axis to spread. We show that each collapse follows a self-similar form given by $E \sim(Z-z)^{-1 / 3}, r \sim(Z-z)^{2 / 3}$, and $\tau-\tau^{*}(z) \sim(Z-z)^{1 / 3}$, where $Z$ is the blowup point and $\tau^{*}(z)$ follows the maximum of $E_{0}$.

We also point out that there is a distinct difference in collapse behavior between the linearly and azimuthally polarized cases but we leave it to a later paper [8] to report details.

Finally, we briefly describe how, in the case of strong longitudinal dispersion, a much weaker nonlinearity, and an almost monochromatic rather than broadband pulse, (1) takes on nonlinear Schrödinger form with normal $(B>0)$ and anomalous $(B<0)$ longitudinal dispersion. In that case, the behavior of the electric field envelope follows that discussed by Luther et al. [7].

\section{Derivation of RUPPE and MKPI}

To make the presentation as simple as possible and to postpone approximations until absolutely necessary, we start with an azimuthally polarized field $E=\hat{\theta} E(r, z, t)$, where $\hat{\theta}$ is the unit vector in the angular direction and $z$ is the direction of propagation. The result we obtain for a linearly polarized wave $\vec{E}=\hat{e} E_{0}(x, y, z, t)+\cdots$ will be almost the same except that it is necessary to include a small electric field component in the propagation direction $z$ in order to satisfy the divergence free condition. In addition, the diffraction 
operator will be the Laplacian $\partial^{2} / \partial r^{2}+(1 / r)(\partial / \partial r)$ rather than $\delta^{2}=\partial^{2} / \partial r^{2}+(1 / r)(\partial / \partial r)-1 / r^{2}$.

We take as constitutive relations $\vec{B}=\mu \vec{H}$ and

$$
\begin{aligned}
\vec{D}=\varepsilon_{0} \hat{\theta}( & E+\int_{-\infty}^{t} \chi(t-\tau) E(\tau) d \tau+\int_{-\infty}^{t} \chi^{(3)} \\
& \left.\times\left(t-\tau_{1}, t-\tau_{2}, t-\tau_{3}\right) E\left(\tau_{1}\right) E\left(\tau_{2}\right) E\left(\tau_{3}\right) d \tau_{1} d \tau_{2} d \tau_{3}\right) .
\end{aligned}
$$

We write the Fourier transforms of $E(r, z, t)$, the linear and nonlinear susceptibility $\chi(t)$ and $\chi^{(3)}\left(t_{1}, t_{2}, t_{3}\right)$ to be $e(r, z, \omega)=\int_{-\infty} E(r, z, t) e^{i \omega t} d t, \hat{\chi}(\omega)$ and $\hat{\chi}^{(3)}\left(\omega_{1}, \omega_{2}, \omega_{3}\right)$, respectively. From Maxwell's equation $\nabla \times \nabla \times \vec{E}=$ $\mu\left(\partial^{2} \vec{D} / \partial t^{2}\right)$ and $\mu \varepsilon_{0}=1 / c^{2}$, we obtain the exact relation

$$
\begin{aligned}
& \frac{\partial^{2} e}{\partial z^{2}}+\delta^{2} e+\frac{\omega^{2}}{c^{2}}(1+\hat{\chi}(\omega)) e \\
& =-\frac{2 \pi \omega^{2}}{c^{2}} \int \chi^{(3)}\left(\omega_{1}, \omega_{2}, \omega_{3}\right) e\left(\omega_{1}\right) e\left(\omega_{2}\right) e\left(\omega_{3}\right) \\
& \quad \times \delta\left(\omega-\omega_{1}-\omega_{2}-\omega_{3}\right) d \omega_{1} d \omega_{2} d \omega_{3},
\end{aligned}
$$

where $\delta(x)$ is the Dirac delta function. Anticipating that we will be taking nonlinearity and diffraction to be small when compared to the linear response $\left(n^{2}(\omega) \omega^{2} / c^{2}\right) e, n^{2}(\omega)=1+$ $\hat{\chi}(\omega)$, we write $e(r, z, \omega)$ as the sum of forward and backward fields. Let

$$
e(r, z, \omega)=A(r, z, \omega) e^{i k_{0}(\omega) z}+B(r, z, \omega) e^{-i k_{0}(\omega) z},
$$

where $k_{0}^{2}(\omega)=\left(\omega^{2} / c^{2}\right)\left(1+\hat{\chi}_{r}(\omega)\right), \hat{\chi}_{r}(\omega)$ the real part of $\hat{\chi}(\omega)$ and make the free choice of a relation between $A$ and $B$ to be $(\partial A / \partial z) e^{i k_{0}(\omega) z}+(\partial B / \partial z) e^{-i k_{0}(\omega) z}=0$ (cf. method of variation of parameters). Then substituting (5) into (4), and adding and subtracting the free choice, we obtain the exact relations:

$$
\begin{aligned}
2 i k_{0}(\omega) \frac{\partial A}{\partial z}= & -\frac{i \omega^{2}}{c^{2}} \hat{\chi}_{i}(\omega) A-\frac{i \omega^{2}}{c^{2}} \hat{\chi}_{i}(\omega) B e^{-2 i k_{0}(\omega) z} \\
& -\delta^{2} A-\delta^{2} B e^{-2 i k_{0}(\omega) z}-\frac{2 \pi}{c^{2}} \omega^{2} P e^{-i k_{0}(\omega) z}
\end{aligned}
$$

where

$$
\begin{aligned}
P= & \int \chi^{(3)}\left(\omega_{1}, \omega_{2}, \omega_{3}\right) \delta\left(\omega-\omega_{1}-\omega_{2}-\omega_{3}\right) d \omega_{1} d \omega_{2} d \omega_{3} \\
& \times\left(A\left(\omega_{1}\right) e^{-i k_{0}\left(\omega_{1}\right) z}+B\left(\omega_{1}\right) e^{-i k_{0}\left(\omega_{1}\right) z}\right) \\
& \times\left(\omega_{1} \longrightarrow \omega_{2}\right)\left(\omega_{1} \longrightarrow \omega_{3}\right),
\end{aligned}
$$

and an equivalent equation for $\partial B / \partial z$. These equations are exact. There have been no approximations thus far. Note that the parameters $A$ and $B$ introduced here and discussed in the following are different from those introduced in (2).

We will now ask how can we approximate solutions to (6), and (7) if their right hand sides are small? Suppose we set $A=A_{0}+A_{1}+\cdots, B=B_{0}+B_{1}+\cdots$. To leading order, $A_{0}$ and $B_{0}$ will be independent of $z$ but, in order to remove secular terms (terms growing as $z$ ) in the iterates $A_{1}, B_{1}, \ldots$, we will have to choose their slow variations $\partial A_{0} / \partial z$ and $\partial B_{0} / \partial z$ accordingly. So the first task is to identify the secular terms in $A_{1}$ and $B_{1}$. The equation for $A_{1}$ is

$$
\begin{aligned}
2 i k_{0}(\omega) \frac{\partial A_{1}}{\partial z}= & -\frac{i \omega^{2}}{c^{2}} \hat{\chi}_{i}(\omega) A_{0}-\frac{i \omega^{2}}{c^{2}} \hat{\chi}_{i}(\omega) B_{0} e^{-2 i k_{0}(\omega) z} \\
& -\delta^{2} A_{0}-\delta^{2} B_{0} e^{-2 i k_{0}(\omega) z}+P_{0} e^{-i k_{0}(\omega) z},
\end{aligned}
$$

where $P_{0}$ is $P$ given in (7) with $A$ replaced by $A_{0}$. Since, to leading order, $A_{0}$ and $B_{0}$ are $z$ independent, it is clear that by direct integration from $z=0$ to $z=z$, the first and third terms on the right hand side of (8) are secular whereas the second and fourth, each of whose fast dependence is $\left(\left(e^{-i k_{0}(\omega) z}-1\right) / i k_{0}(\omega)\right)$, are not. The more interesting discussion involves the nonlinear term one member of which, when integrated from 0 to $z$, is

$$
\begin{aligned}
& \int \chi^{(3)}\left(\omega_{1}, \omega_{2}, \omega_{3}\right) A_{0}\left(\omega_{1}\right) A_{0}\left(\omega_{2}\right) A_{0}\left(\omega_{3}\right) \\
& \quad \times\left(\frac{e^{i\left(k_{0}\left(\omega_{1}\right)+k_{0}\left(\omega_{2}\right)+k_{0}\left(\omega_{3}\right)-k_{0}(\omega)\right) z}-1}{i\left(k_{0}\left(\omega_{1}\right)+k_{0}\left(\omega_{2}\right)+k_{0}\left(\omega_{3}\right)-k_{0}(\omega)\right)}\right) \\
& \quad \times \delta\left(\omega-\omega_{1}-\omega_{2}-\omega_{3}\right) d \omega_{1} d \omega_{2} d \omega_{3} .
\end{aligned}
$$

In order to assess whether this term gives rise to secular behavior, we must ask what is its limiting behavior as $z$ becomes large. To answer this, we require a little mathematics. We state two important results. If $f(x)$ is an ordinary (measurable) function which is absolutely integrable and $h(x)$ is not identically zero over a finite interval in the domain of integration, we know that $\lim _{z \rightarrow \infty} \int f(x) \exp (i h(x) z) d x=0$ (the RiemannLebesgue lemma) and that $\lim _{z \rightarrow \infty} \int f(x) \Delta(h(x)) d x=$ $\int \pi \operatorname{sgn} z \delta(h(x))+i P(1 / h(x)) f(x) d x$ where $\Delta(h)=\left(e^{i h z}-\right.$ $1) / i h$ and $P$ denotes the Cauchy Principal Value. This means that as long as the amplitudes $A_{0}(\omega), B_{0}(\omega)$ are ordinary (as opposed to, say, Dirac delta) functions and are absolutely integrable, the nonlinear terms will give a bounded contribution to $A_{1}$ and be therefore nonsecular unless $h\left(\omega_{1}, \omega_{2}, \omega_{3}\right)=k_{0}\left(\omega_{1}\right)+k_{0}\left(\omega_{2}\right)+k_{0}\left(\omega_{3}\right)-k_{0}(\omega)$ is identically zero over finite regions of $\omega_{2}, \omega_{3}$ space (note: $\left.\omega_{1}=\omega-\omega_{2}-\omega_{3}\right)$. Namely, the nonlinear term will give no cumulative long distance effect unless $h$ is identically zero. This occurs only when there is no (at least to leading order) longitudinal dispersion.

This realization makes the propagation of narrow pulses with broadband spectrum for which the Fourier transforms are smooth very different from that of almost monochromatic pulses for which the Fourier amplitudes $A_{0}(\omega)$ and $B_{0}(\omega)$ are close to Dirac delta functions centered on some finite set of carrier frequencies. In the latter case, the nonlinear term can be secular either because of modal interactions leading to a nonlinear Schrödinger type nonlinearity or resonant interactions leading to coupled wave packets. Namely, if the amplitudes $A_{0}(\omega)$ can be Dirac delta functions, the integral (9) becomes a sum and $\Delta(h)$ will be equal to $z$ at any points in $\omega_{1}, \omega_{2}, \omega_{3}$ space with $\omega_{1}+\omega_{2}+\omega_{3}=\omega$ where the 
resonant condition $h \equiv k_{0}\left(\omega_{1}\right)+k_{0}\left(\omega_{2}\right)+k_{0}\left(\omega_{3}\right)-k_{0}(\omega)=0$ holds. We return to this situation at the end of this section. For now, we focus on the case of narrow pulses for which the Fourier transforms $e(r, z, \omega)$ are smooth in $\omega$ and for which (9) is only secular when $k_{0}\left(\omega_{1}\right)+k_{0}\left(\omega_{2}\right)+k_{0}\left(\omega_{3}\right)-k_{0}(\omega)$ is (almost) identically zero; namely, when $k_{0}(\omega)=n_{0}(\omega / c)$ for constant $n_{0}=\sqrt{1+\hat{\chi}_{r}}$. It should now also be clear that any of the other products in $P_{0}$ such as those involving $A_{0}\left(\omega_{1}\right) A_{0}\left(\omega_{2}\right) B_{0}\left(\omega_{3}\right)$ with fast $z$ behavior $\Delta\left(k_{0}\left(\omega_{1}\right)+k_{0}\left(\omega_{2}\right)-\right.$ $\left.k_{0}\left(\omega_{3}\right)-k_{0}(\omega)\right)$ never give rise to secular terms. Thus, the interaction between right and left going pulses is small and does not affect their deformations.

We now return to (4) and write $\hat{\chi}(\omega)$ as $\hat{\chi}(\omega)-\hat{\chi}(0)+$ $\hat{\chi}(0)$ and assume that $\hat{\chi}(\omega)-\hat{\chi}(0)$ is small compared to $\hat{\chi}(0)$. This small difference will give rise to weak dispersion and attenuation. We recall that since $E(r, z, t)$ and $D(r, z, t)$ are real, $\hat{\chi}(-\omega)=\hat{\chi}^{*}(\omega)$ so that $\hat{\chi}_{r}(\omega)$ is even in $\omega$ and $\hat{\chi}_{i}(\omega)$ is odd in $\omega$. We will then obtain an equation (6) for $A(r, z, \omega)$ in which $k_{0}(\omega)=n_{0}(\omega / c), n_{0}=\sqrt{1+\hat{\chi}_{r}(0)}$ and the susceptibility deviation from $\hat{\chi}(0)$ will manifest itself as the term $\left((\hat{\chi}(0)-\hat{\chi}(\omega)) / c^{2}\right) \omega^{2} A$ on the right hand side of (6). When we now repeat the analysis, we find the only secular terms arising in $A_{1}$ are those for which all fast dependence on $z$ have been removed. To suppress these secular terms in $A_{1}$, we allow $A_{0}$ to be slowly varying. We find the equation we call RUPPE,

$$
\begin{aligned}
& 2 i n_{0} \frac{\omega}{c} \frac{\partial A_{0}}{\partial z} \\
&= \frac{\hat{\chi}(0)-\hat{\chi}(\omega)}{c^{2}} \omega^{2} A_{0}-\delta^{2} A_{0} \\
&-\frac{2 \pi \omega^{2}}{c^{2}} \int \chi^{(3)}\left(\omega_{1}, \omega_{2}, \omega_{3}\right) A_{0}\left(\omega_{1}\right) A_{0}\left(\omega_{2}\right) A_{0}\left(\omega_{3}\right) \\
& \quad \times \delta\left(\omega-\omega_{1}-\omega_{2}-\omega_{3}\right) d \omega_{1} d \omega_{2} d \omega_{3} .
\end{aligned}
$$

It says, in effect, that the evolution of the right going Fourier amplitude of the electric field in frequency space travels without deformation on distances of the order of many wavelengths but is distorted over much longer distances by a combination of dispersion and attenuation, diffraction and nonlinearity. It has several very important properties.

2.1. Analogue of the Turitsyn-Falkovich Theorem. In its simplest form, we take $\hat{\chi}^{(3)}\left(\omega_{1}, \omega_{2}, \omega_{3}\right)$ constant and ignore $(\hat{\chi}(0)-\hat{\chi}(\omega))$. We define the flux, variance, current, and Hamiltonian to be
(a) $F(z)=\int_{z}^{\infty} A_{0} A_{0}^{*} r d r d \omega$,
(b) $V(z)=\int_{0}^{\infty} r^{2} A_{0} A_{0}^{*} r d r d \omega$,
(c) $C(z)=2 i \int_{0}^{\infty} \frac{r}{\omega}\left(A_{0} \frac{\partial A_{0}^{*}}{\partial r}-A_{0}^{*} \frac{\partial A_{0}}{\partial r}\right) r d r d \omega$,

$$
\text { (d) } \begin{aligned}
H(z)=\delta \int r d r d \omega & \frac{1}{\omega^{2}}\left(\frac{\partial A_{0}}{\partial r}+\frac{1}{r} A_{0}\right)\left(\frac{\partial A_{0}^{*}}{\partial r}+\frac{1}{r} A_{0}^{*}\right) \\
-\frac{8 \pi}{c^{2}} \int & r d r d \omega d \omega_{1} d \omega_{2} d \omega_{3} \\
& \times A_{0}(\omega) A_{0}\left(\omega_{1}\right) A_{0}\left(\omega_{2}\right) A_{0}\left(\omega_{3}\right) \\
& \times \delta\left(\omega+\omega_{1}+\omega_{2}+\omega_{3}\right),
\end{aligned}
$$

where $A_{0}(r, z, \omega)$ tends to zero at $r=0$ and $r=\infty$.

The azimuthally polarized case demands that the electric field be zero at $r=0$ in order that the magnetic field is zero (strictly speaking, the magnetic field will be very weak and longitudinally polarized but has no azimuthal component.) Then, we can easily show

$$
\frac{d F}{d z}=0, \quad \frac{d V}{d z}=C, \quad \frac{d C}{d z}=H, \quad \frac{d H}{d z}=0 .
$$

Thus, $d^{2} V / d z^{2}=H$, and if the constant (in $z$ ) $H$ is negative at $z=0$, then the positive definite variance $V$ would become negative in a finite distance $z$. The only conclusion is that $A_{0}(r, z, \omega)$ must develop a singularity before then.

If we include dispersion and ignore attenuation, then for $\hat{\chi}(0)-\hat{\chi}(\omega)<0$, the case of anomalous dispersion, a similar conclusion holds. For normal dispersion, we will shortly see that a different kind of singularity occurs. It is not known for which class of nonconstant nonlinear susceptibilities $\chi^{(3)}\left(\omega_{1}, \omega_{2}, \omega_{3}\right)$, singularities must form.

2.2. The MKPI Equation. The facts that the forward and backward going components can be separated and that $\hat{\chi}(\omega)$ is almost constant suggest that we rewrite (10) as an equation for the leading order component of the electric field

$$
E_{0}\left(r, z, \tau=t-\frac{n_{0} z}{c}\right)=\frac{1}{2 \pi} \int A_{0}(r, z, \omega) e^{-i \omega\left(t-\left(n_{0} z / c\right)\right)} d \omega .
$$

We note that $-i \omega A_{0}$ has its Fourier transform $\partial E_{0} / \partial \tau$. Then if we take $\hat{\chi}^{(3)}$ constant, (10) becomes

$$
\frac{2 n_{0}}{c} \frac{\partial^{2} E_{0}}{\partial \tau \partial z}+\frac{2 \pi \hat{\chi}^{(3)}}{c^{2}} \frac{\partial^{2} E_{0}^{3}}{\partial \tau^{2}}+F T\left\{\frac{\hat{\chi}(0)-\hat{\chi}(\omega)}{c^{2}} \omega^{2} A_{0}\right\}=\delta^{2} E_{0} .
$$

If we further approximate $\hat{\chi}(0)-\hat{\chi}(\omega)=-i A \omega t_{0}-B \omega^{2} t_{0}^{2}$, $A, B$ dimensionless, which is not unreasonable over a large frequency range for air, then (14) becomes

$$
\frac{\partial}{\partial \tau}\left(\frac{\partial E_{0}}{\partial z}+\frac{3 \pi \hat{\chi}^{(3)} E_{0}^{2}}{n_{0} c} \frac{\partial E_{0}}{\partial \tau}-\frac{B t_{0}^{2}}{2 n_{0} c} \frac{\partial^{3} E_{0}}{\partial \tau^{3}}-\frac{A t_{0}}{2 n_{0} c} \frac{\partial^{2} E_{0}}{\partial \tau^{2}}\right)=\frac{c}{2 n_{0}} \delta^{2} E_{0} .
$$

We introduce the nondimensional scalings

$$
E_{0} \longrightarrow e_{0} E_{0}, \quad \tau \longrightarrow t_{0} \tau, \quad z \longrightarrow z_{0} z, \quad x, y \longrightarrow L(x, y)
$$


and define

$$
\begin{aligned}
z_{\mathrm{NL}}=\frac{n_{0} c t_{0}}{3 \pi \hat{\chi}^{(3)} e_{0}^{2}}, \quad z_{\text {diff }} & =\frac{2 n_{0} L^{2}}{c t_{0}}, \quad z_{\text {disp }}=\frac{2 n_{0} c t_{0}}{|B|}, \\
z_{\text {att }} & =\frac{2 n_{0} c t_{0}}{A},
\end{aligned}
$$

then (15) is $(s=\operatorname{sgn} B)$

$$
\frac{\partial}{\partial \tau}\left(\frac{\partial E_{0}}{\partial z}+\frac{z_{0}}{z_{\mathrm{NL}}} E_{0}^{2} \frac{\partial E_{0}}{\partial \tau}-\frac{s z_{0}}{z_{\text {disp }}} \frac{\partial^{3} E_{0}}{\partial \tau^{3}}-\frac{z_{0}}{z_{\text {att }}} \frac{\partial^{2} E_{0}}{\partial \tau^{2}}\right)=\frac{z_{0}}{z_{\text {diff }}} \delta^{2} E_{0}
$$

which is the modified Kadomtsev-Petviashvili equation of type 1. If we include a plasma oscillation to account for low-frequency behavior, this is exactly the equation used by BLMS [1] and derived in other contexts by Kuznetsov and collaborators in the nineteen eighties. It is also connected with the work of both Alterman and Rauch [4] and Schaffer and Wayne [5] who realized that, to leading order, the propagation characteristics of short pulses are captured by looking at the deformation of the right going Riemann invariant $E_{0}$.

Depending on which effect is dominant, we will choose $z_{0}$ accordingly. For air, for pulses of dimensionless power $n_{2} I\left(e=\left(3 / 8 n_{0}\right) \chi^{(3)} e_{0}^{2}\right)$ of about $10^{-4}$, and for $c t_{0} \sim 10 \mu \mathrm{m}$, for beam widths of between $100 \mu \mathrm{m}$ and $1 \mathrm{~mm}$, the nonlinear and diffraction distances are of the order of $10 \mathrm{~mm}$, the dispersion length about $100 \mathrm{~mm}$. Attenuation is negligibly small. In this case, we choose $z_{0}=z_{\mathrm{NL}}$.

The behaviors in the two cases $s=1$ and $s=-1$ corresponding to normal and anomalous dispersion are markedly different. For normal dispersion, as BLMS have shown, the forward slope (in $\tau$; rear part in a fixed frame) steepens. Before it becomes multivalued, it is arrested by an outburst of regularizing oscillations. The BLMS analysis of the onset of this singular behavior captures the beginning of this behavior. The dimensional frequency range of these waves is given by $\Omega \sim\left(1 / t_{0}\right) \sqrt{z_{\text {disp }} / z_{\mathrm{NL}}}$. These waves, with wavevector $\vec{k}_{0}=\left(0,0, k_{0}(\Omega)\right)$, pick up resonant four wave partners $\vec{k}_{ \pm}=\left( \pm \vec{k}_{1}, k_{0}(\Omega)\right)$ via the resonance $2 \vec{k}_{0}=\vec{k}_{+}+\vec{k}_{-}$ and eventually disperse the energy transversely. The initial behavior is captured by the scaling $\tau-\left(\rho_{z} / 4 \rho\right) r^{2}$ variable in BLMS (see their equation (29)).

This behavior is reminiscent of what happens for the three-dimensional nonlinear Schrödinger equation with a self-focusing nonlinearity, diffraction, and normal dispersion in the propagation direction. There, if normal dispersion is initially weak, the cross sectional part of the pulse with the greatest power begins to focus into a collapse as a result of the balance between diffraction and self focusing nonlinearity. By following the evolution in $z$, the propagation direction, of the self-similar scaling variables describing the collapse, we see the effect of normal dispersion is to arrest the collapse and spread the energy of the failed collapse via four-wave resonant interactions.

For anomalous dispersion, the behavior is very different. For linearly polarized waves, the electric field concentrates in the vicinity of $r=0, z=Z$ at a retarded time $\tau=\tau^{*}(Z)$. For azimuthally polarized waves, the energy concentrates in a collapsing ring about $r=0$. In the following section, we show the results of simulations in the normal and anomalous dispersion cases.

As the final remark of this section, we briefly describe what happens to an almost monochromatic pulse in the case where dispersion is strong, namely, where $z_{\mathrm{NL}} \gg z_{\text {disp. }}$. In that case, the envelope $C(x, y, z, \tau)$ of a carrier wave $\exp \left(-i \omega_{0} \tau+i k_{0}\left(\omega_{0}\right) z\right)$ will evolve along its group velocity trajectory and deform under the joint action of a nonlinear Schrödinger nonlinearity $C^{2} C^{*}$, diffraction $\left(\partial^{2} k_{z} / \partial k_{x^{2}}\right) \nabla^{2} C$, and dispersion $\left(\partial^{2} k z / \partial \omega^{2}\right)\left(\delta^{2} C / \delta \tau^{2}\right)$.

A natural question to ask is: if, in a medium which contains a strong resonant band about $\omega \sim \omega_{0}$, is it possible that an initially broad band pulse can peel off an almost monochromatic wave packet which separates from the main pulse and whose further propagation behavior is determined by a nonlinear Schrödinger type equation describing its deformation due to dispersion and nonlinearity along its local group velocity trajectory $t=k_{0}^{\prime}\left(\omega_{0}\right) z$ ?

\section{Numerical Study of the MKPI Equation}

With the choice $z_{0}=z_{\mathrm{NL}}$, equation (18) can be written

$$
\frac{\partial}{\partial \tau}\left(\frac{\partial E_{0}}{\partial z}+\frac{\partial E_{0}^{3}}{\partial \tau}-B \frac{\partial^{3} E_{0}}{\partial \tau^{3}}-A \frac{\partial^{2} E_{0}}{\partial \tau^{2}}\right)=D \Delta_{\perp} E_{0}
$$

where

$$
B=\frac{z_{\mathrm{NL}}}{z_{\text {disp }}}, \quad A=\frac{z_{\mathrm{NL}}}{z_{\mathrm{att}}}, \quad D=\frac{z_{\mathrm{NL}}}{z_{\text {diff }}} .
$$

The discussion will be limited to the case of plane polarization $\Delta_{\perp}=\partial^{2} / \partial x^{2}+\partial^{2} / \partial y^{2}$.

The focus of this section is to study the qualitative aspects of (19), with particular attention on the role of dispersion in preventing or promoting singular behavior. It is notable that for the case of one transverse dimension $\left(\Delta_{\perp}=\partial^{2} / \partial y^{2}\right)$, much is already understood about the MKP equation (see e.g., Klein and Saut [9]). To our knowledge, the only detailed study of the three dimensional case in the nonlinear optics context was conducted by Balakin et al. [1]. We will compare their findings to ours below.

Numerical simulations are used to illustrate the behavior of solutions of (19). Our method is fairly standard, employing spectral (Fourier and Hankel transform) representations of $\tau$ and $r$ derivatives. Approximation of the $z$-derivative employs fourth-order exponential time differencing $[10,11]$. The computational domain is periodic in $\tau$, which has the effect of cutting off small, physically irrelevant frequencies.

Our main attention will focus on the effects of large frequency dispersion encoded in the parameter $B$. To this end we fix $A=0$ and $D=1$, and assume radial symmetry $\left(\Delta_{\perp} E_{0}=r^{-1}\left(r\left[E_{0}\right]_{r}\right)_{r}\right)$. It is particularly instructive to compare normal $(B>0)$ to anomalous $(B<0)$ dispersion. While the former is expected for optical pulses, the latter case demonstrates the significance of high frequency dispersion and the need for normal dispersion as a regularizing mechanism. 


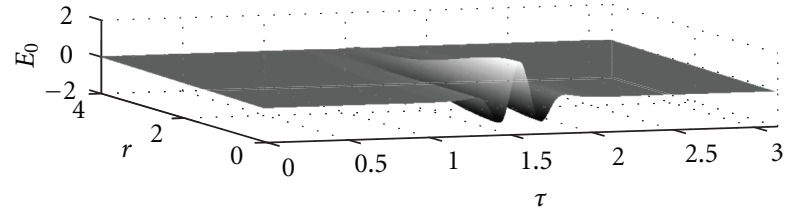

(a)

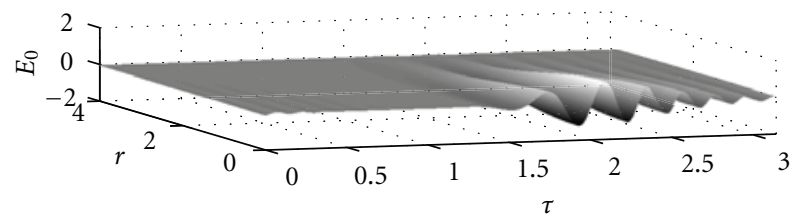

(b)

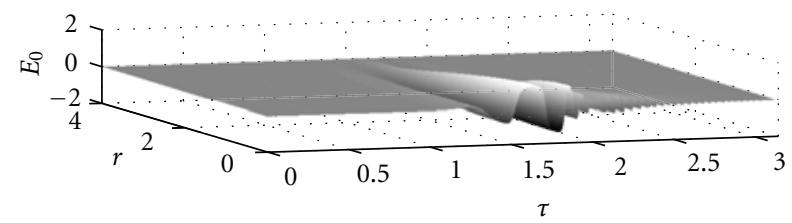

(c)

Figure 1: Evolution of a narrow pulse (a) for large $(B=0.1$, (b) and small $(B=0.0001,(\mathrm{c}))$ normal dispersion at early stages $(z=$ 0.2 ). In the latter case, the profile steepens, corresponding to the generation of higher frequency components.

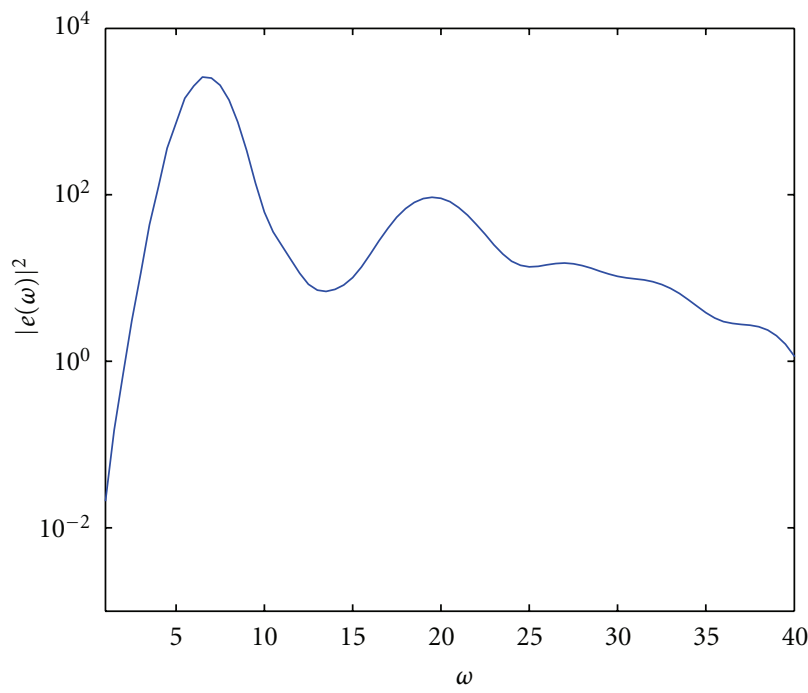

FIGURE 2: Spectral content of a pulse at early stages of evolution, showing generation of third harmonics $(B=0.0001) . e(\omega)$ is the Fourier transform of the centerline profile $E_{0}(r=0)$.

3.1. Normal Dispersion. We consider initial data corresponding to a short pulse of unit width

$$
E_{0}(r, z=0, \tau)=\exp \left(-r^{2}\right) f(\tau)
$$

where $f$ has Fourier transform $f_{0} \exp \left(-(\omega-2 \pi)^{2}\right)$ and $f_{0}$ is chosen so that the maximum amplitude of $E_{0}(r, z=0, \tau)$ is equal to one.

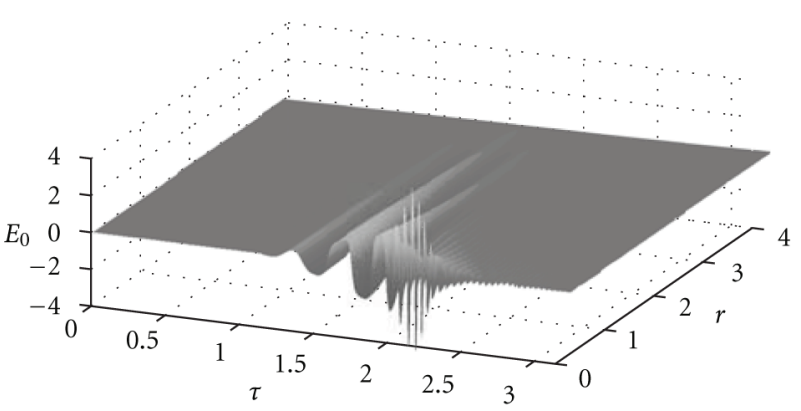

FIGURE 3: Self-focusing of a pulse for small normal dispersion $(B=$ $0.0001)$ when $z=1$. Compression in the transverse direction is apparent in the back ( $\tau$ large) of the pulse.

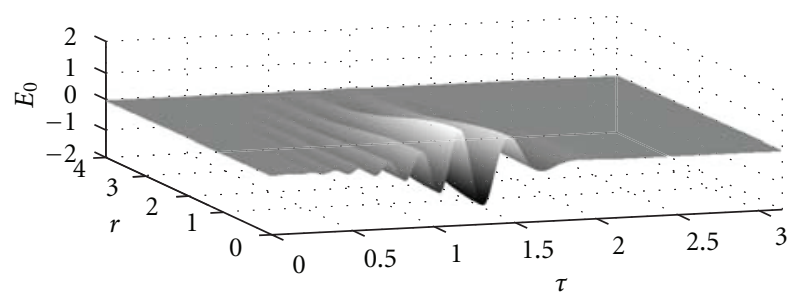

(a)

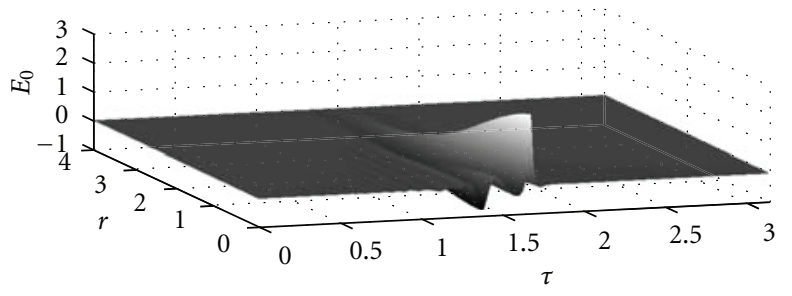

(b)

FIGURE 4: Evolution of a pulse for large $(B=-1$, (a)) and small ( $B=-0.001$, (b) ) anomalous dispersion. In the latter case, there is compression both temporally and in the transverse direction. The bottom figure shows an incipient collapse event that will develop into a singularity with infinite amplitude.

Consider first the case of large normal dispersion $B=0.1$. In this situation, the pulse does not retain its structure, but rather disintegrates into a wave train dominated by a small band of frequencies (Figure 1, middle).

When the effect of dispersion is smaller $(B=0.0001)$, one observes steepening of the pulse (Figure 1(c)). This coincides with generation of harmonics by four-wave interactions (Figure 2). By comparing the effective size of nonlinear and dispersive terms, one sees that formation of a shock will be arrested when steepening reaches a dimensional timescale $\tau_{\max } \approx t_{0} B^{-1 / 2}$. By virtue of $B$ depending inversely on initial amplitude, it follows that stronger pulses generate higher frequencies.

At a propagation distance comparable to $z_{0}$, self-focusing is apparent (Figure 3). We observe that compression in the transverse direction is stronger when normal dispersion is smaller. This might be expected, since normal dispersion is 


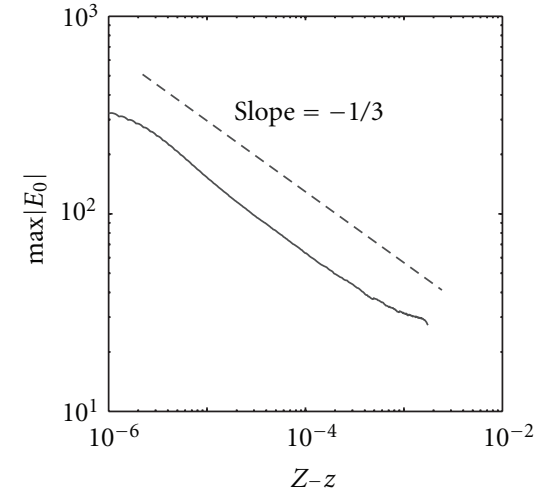

(a)

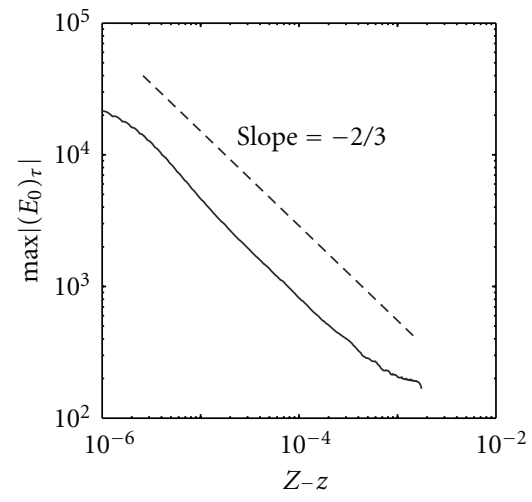

(b)

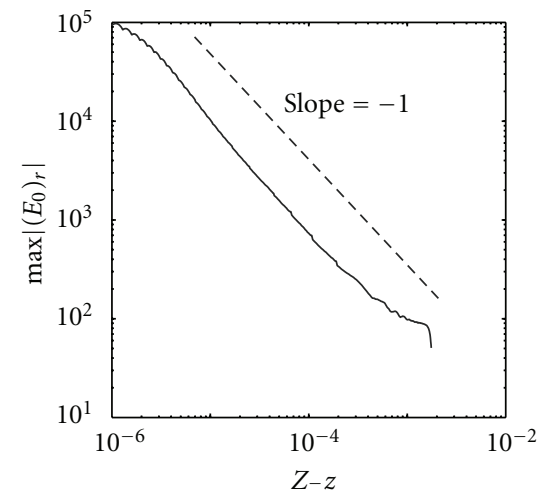

(c)

Figure 5: The maximum amplitude of $E_{0}$ and its derivative during the collapse. The results are consistent with the power law behavior (22) derived in the text.

known to prevent self-focusing collapse in the monochromatic limit described by the nonlinear Schrödinger equation [7].

3.2. Anomalous Dispersion and Singular Collapse. The case $B<0$ was also studied using the same initial data. This is expected to be different since finite time singularities may occur. In analogy to the focusing nonlinear Schrödinger equation, one can show this by use of a virial-type argument $[1,3]$.

When $B=-1$, no singular behavior is observed. Rather, breakup of the pulse into a wave train occurs much like the large normal dispersion case. (Figure 4(a)). This is analogous to the situation in the nonlinear Schrödinger equation where solutions do not have sufficient power for collapse.

In contrast, when $B=-0.001$, the pulse collapses (Figure $4(\mathrm{~b}))$ in both $\tau$ and $r$, and the amplitude grows without bound. This occurs at a point where $\left(E_{0}\right)_{\tau}$ is large, suggesting that blowup is initiated when dispersive effects become significant.

The quantitative aspects of this singularity can be analyzed by assuming that scales behave as power laws

$$
E_{0} \sim(Z-z)^{\alpha}, \quad \tau \sim(Z-z)^{\beta}, \quad r \sim(Z-z)^{\gamma},
$$

where $z=Z$ is the blowup point. Balancing dispersion, nonlinearity and diffractive effects for $z \rightarrow Z$ requires

$$
\alpha-\beta-1=3 \alpha-2 \beta=\alpha-4 \beta=\alpha-2 \gamma,
$$

which means $\alpha=-1 / 3, \beta=1 / 3, \gamma=2 / 3$. These scalings were confirmed numerically (Figure 5 ).

Numerical evidence also suggests that collapse occurs in a universal, self-similar fashion (Figure 6). One may derive an equation for the profile by using the ansatz

$$
\begin{gathered}
E_{0}=(Z-z)^{-1 / 3} U(\xi, \eta), \quad \xi=(Z-z)^{-1 / 3}\left(\tau-\tau^{*}(z)\right), \\
\eta=(Z-z)^{-2 / 3} r
\end{gathered}
$$

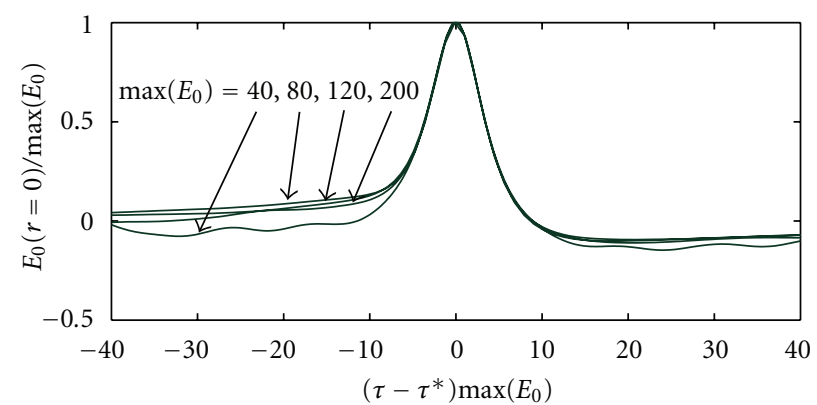

FIGURE 6: Scaled profiles of $E_{0}$ along the centerline $\eta=(Z-$ $z)^{-2 / 3} r=0$, showing collapse onto a single self-similar profile. Similar results hold for $\eta \neq 0$.

where the blowup point is at $(\tau, r, z)=\left(\tau^{*}(Z), 0, Z\right)$. Inserting (24) into (19) gives the similarity equation (ignoring the attenuation term)

$$
\left(\frac{1}{3}\left[U+\xi U_{\xi}+2 \eta U_{\eta}\right]+U^{2} U_{\xi}+B U_{\xi \xi \xi}\right)_{\xi}-\frac{D}{\eta}\left(\eta U_{\eta}\right)_{\eta}=0 .
$$

Properties of (25) are still under investigation.

3.3. Shock Formation Versus Blowup. Our numerical findings indicate two types of singular or nearly singular behavior. The first type is characterized by steepening of the pulse front. Normal dispersion always appears to arrest steepening that would lead to a shock-type solution. The other candidate for singular behavior is blowup, that is, unbounded growth in amplitude. This appears to only occur for anomalous dispersion.

Balakin et al. [1] make a theoretical prediction of the nature of collapse in (19). The crux of their argument is that dispersion at high frequencies (represented by our $B$ term) can be ignored as the pulse amplitude grows. This leads to 
the assertion that the dominant behavior is described by the equation $(\xi, \theta$ are modified coordinates [1])

$$
w_{\xi}+3 w^{2} w_{\theta}=0
$$

They conclude that shock formation should preempt collapse.

In contrast, we find that unless dispersion is exactly zero, there is no circumstance which leads to shock formation. We note that there is an inconsistency in the argument that leads to (26). During such an event, $E_{\tau}$ and higher order derivatives grow without bound. This means that the fourthorder dispersive term in (19) should in fact become stronger, not weaker, relative to the size of the nonlinear term. On the other hand, (26) probably does capture the steepening behavior during the early phase of pulse evolution.

\section{Summary and Conclusion}

In this paper, we have derived an ultra-broadband electromagnetic propagator as a limiting form of the UPPE equation of Kolesik and Moloney [6]. The resulting MKPI equation, derived using asymptotic analysis, allows us to isolate two key singularities, namely, blowup and shock formation and their regularization due to weak linear dispersion. Our results are related to and extend those of Balakin et al. [1]. The MKPI equation derived here captures the nonlinear evolution of the full electromagnetic field in contrast to narrowband nonlinear envelope NLSE equation. Consequently, it should be relevant to the generation of higher harmonic waves during filamentation in extended gaseous media. However, many open questions remain to be addressed. For example, in this ultra-broadband limit, the linear dispersion and absorption spectral landscape spans the ultraviolet to far infrared portion of the electromagnetic spectrum and will need to be represented by a more general form than that used here. Additionally, as a spectrum broadens due to nonlinear propagation, it will be necessary to include real absorption (and associated dispersion) features associated with real resonances at both ends of this broad electromagnetic spectrum-it is anticipated that such resonances will provide a novel nonlocal regularization mechanism for the singularities discussed here. Moreover, a switch in sign of the group velocity dispersion from normal to anomalous as such resonance features are traversed suggests the possibility of novel dispersive waves or possibly soliton-like structures being emitted. Furthermore, at the very high field intensities under consideration here, strong field ionization becomes important and coupling to a electron-ion plasma will need to be included. This question is controversial in the current literature, and there are arguments to support "ionization-free" nonlinear saturation of the cubic nonlinearity in the case of ultrashort pulse propagation in air [12].

\section{Acknowledgment}

This work was supported by an Air Force Office of Scientific Research Multidisciplinary University Research Initiative (MURI) Grant FA9550-10-1-0561.

\section{References}

[1] A. A. Balakin, A. G. Litvak, V. A. Mironov, and S. A. Skobelev, "Structural features of the self-action dynamics of ultrashort electromagnetic pulses," Journal of Experimental and Theoretical Physics, vol. 104, no. 3, pp. 363-378, 2007.

[2] E. A. Kuznetsov, S. L. Muske, and A. V. Shafarenko, "Collapse of acoustic waves in media with positive dispersion," JETP Letters, vol. 37, no. 5, pp. 241-247, 1983.

[3] S. K. Turitsyn and E. G. Falkovich, "Stability of of magnetoelastic solitons and self-focusing of sound in antiferromagnets," Soviet Physics-JETP, vol. 62, pp. 146-152, 1985.

[4] D. Alterman and J. Rauch, "Diffractive short pulse asymptotics for nonlinear wave equations," Physics Letters A, vol. 264, no. 5, pp. 390-395, 2000.

[5] T. Schaffer and C. E. Wayne, "Propagation of ultra-short optical pulses in cubic nonlinear media," Physica D, vol. 196, no. 1-2, pp. 90-105, 2004.

[6] M. Kolesik and J. V. Moloney, "Nonlinear optical pulse propagation simulation: from Maxwell's to unidirectional equations," Physical Review E, vol. 70, no. 3, Article ID 036604, 11 pages, 2004.

[7] G. Luther, J. V. Moloney, and A. C. Newell, "The effects of normal dispersion on collapse events," Physica D, vol. 74, no. 1-2, pp. 59-73, 1996.

[8] J. Allen-Flowers and K. G. Glasner, in preparation.

[9] C. Klein and J.-C. Saut, "Numerical study of blow up and stabilityof solutions of gereralized Kadomtsev-petviashvili equations," http://arxiv.org/PS_cache/arxiv/pdf/1010/1010.5510v1 .pdf.

[10] S. M. Cox and P. C. Matthews, "Exponential time differencing for stiff systems," Journal of Computational Physics, vol. 176, no. 2, pp. 430-455, 2002.

[11] A.-K. Kassam and L. N. Trefethen, "Fourth-order timestepping for stiff PDEs," SIAM Journal on Scientific Computing, vol. 26, no. 4, pp. 1214-1233, 2005.

[12] P. Bejot, J. Kasparian, S. Henin et al., "Higher-order kerr terms allow ionization-free filamentation in gases," Physical Review Letters, vol. 104, no. 10, article 103903, 2010. 

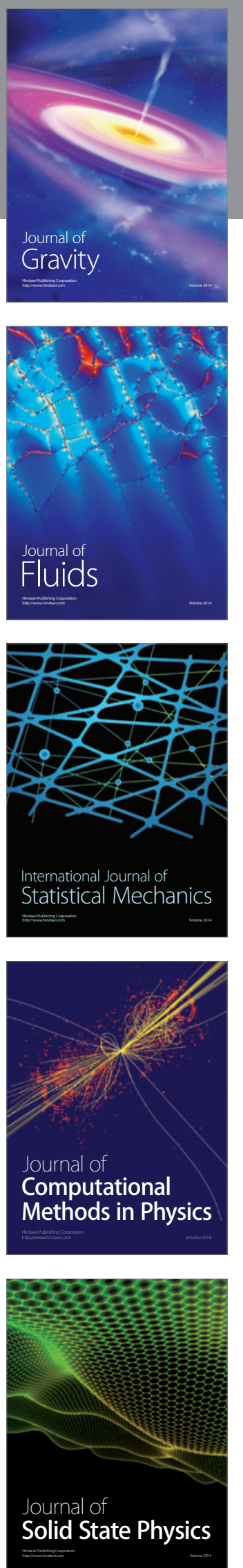

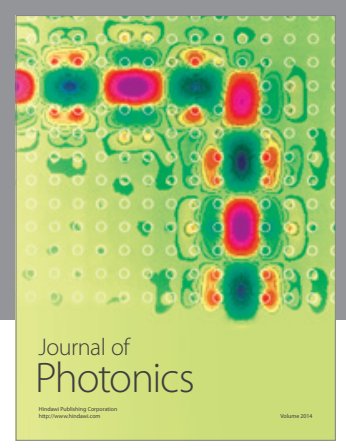

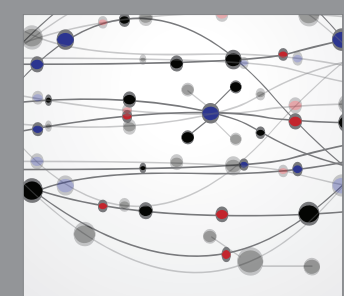

The Scientific World Journal
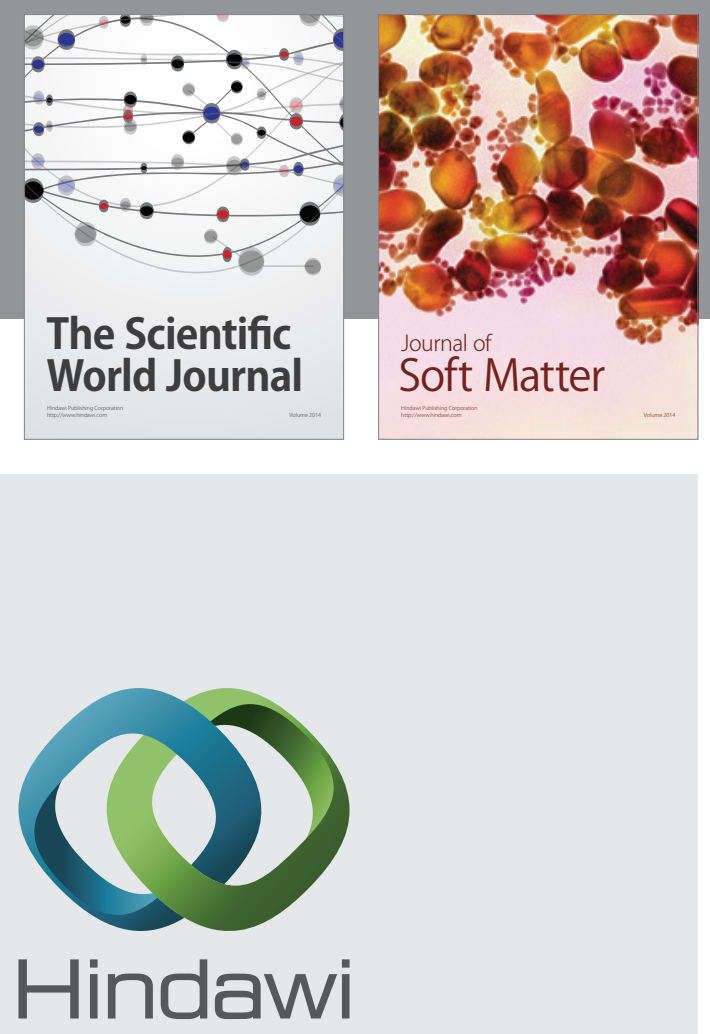

Submit your manuscripts at

http://www.hindawi.com
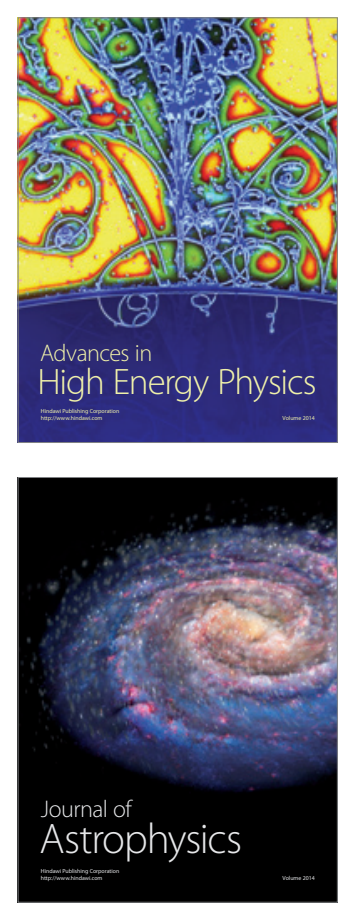
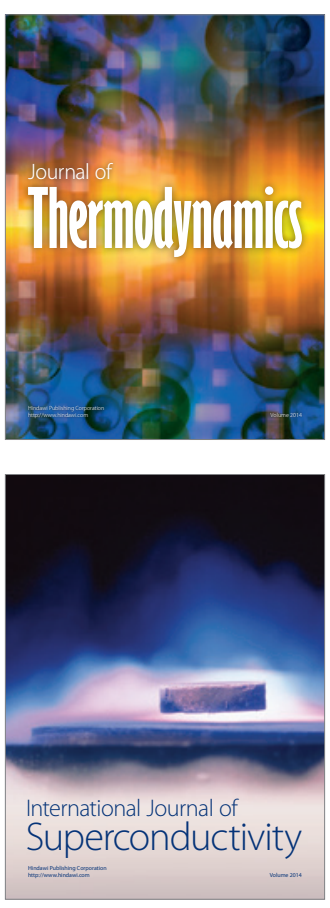
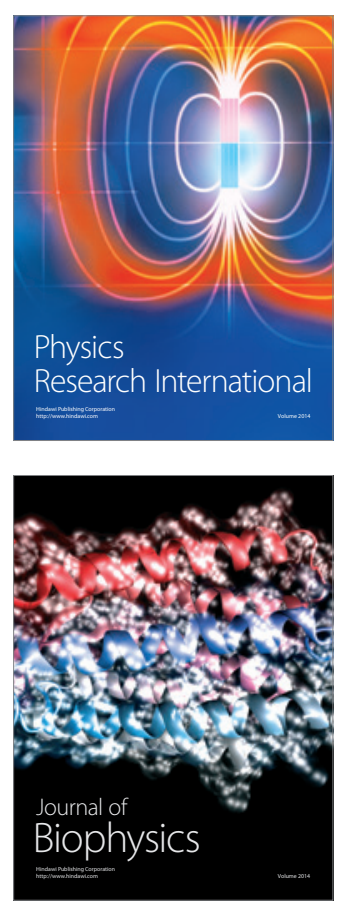
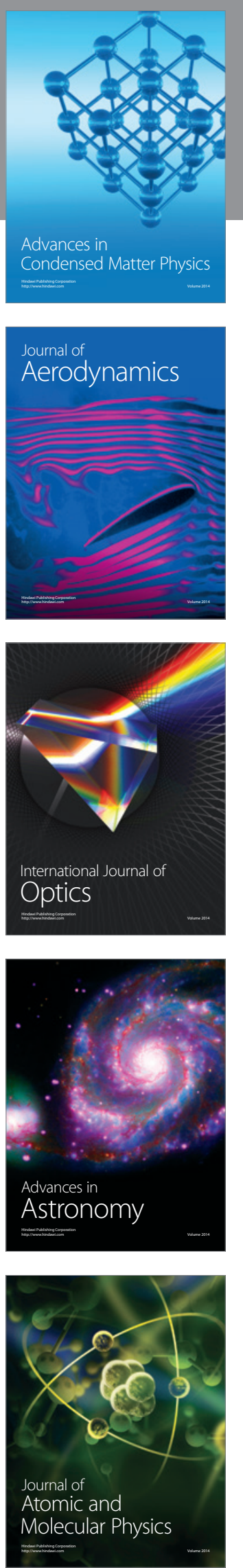